\title{
Fibrous bands in conducting pulmonary arteries
}

\author{
M. TESSA MORRELL AND M. S. DUNNILL \\ From the Nuffield Department of Surgery and Department of Pathology, The Radcliffe \\ Infirmary, Oxford
}

SYNOPSIS Fibrous bands have been described in the elastic (conducting) pulmonary arteries in 38 cases at necropsy, which represents a necropsy incidence of $14.4 \%$. The fibrous bands are considered to represent one of the end results of episodes of pulmonary embolism. The formation of the bands is described and their significance is discussed in relation to their haemodynamic effects. Most of these bands are the result of clinically silent emboli.

During the course of an investigation into the incidence and pathology of pulmonary embolism in hospital patients, the frequent occurrence of fibrous bands, often forming a lace-like network in the pulmonary arteries, was noted. The present paper describes such lesions found in the conducting arteries in 38 cases and discusses the origin and significance of the bands. In a smaller number of cases the lesions were noted in the muscular pulmonary arteries but these cases have not been considered in the following account.

\section{METHODS}

The right lung was taken from 263 cadavers representing a 1 in 5 selection at necropsy on patients who died in the United Oxford Hospitals, where the necropsy rate is $98 \%$. The lungs were all expanded by intrabronchial perfusion with $4 \%$ formaldehyde in saline. They were placed in a bath of fixative for two to six days and cut into $1 \mathrm{~cm}$. thick slices, each of which was examined for arterial lesions. Blocks of all arteries containing pathological lesions were taken for histological examination, being fixed for a further 24 hours in a mercuric chloride formaldehyde saline mixture, dehydrated in alcohol, cleared in chloroform and embedded in paraffin wax. Sections $5 \mu$ thick were cut and stained with haematoxylin and eosin, and by the periodic-acid-Schiff and trichrome methods.

\section{RESULTS}

Thirty-eight cases were found in which there were fibrous bands in the elastic pulmonary arteries. This represented a post-mortem incidence of $14.4 \%$. In 27 of these cases there were more recent thrombi in addition to the fibrous bands. The age incidence of fibrous bands in conducting (elastic) pulmonary arteries at necropsy is shown in the table.

\section{TABLE}

AGE INCIDENCE OF FIBROUS BANDS IN CONDUCTING PULMONARY ARTERIES FOUND AT NECROPSY

\begin{tabular}{ccc} 
Age in Years & Number of Cases & Percentage of Total Cases \\
\hline $40-49$ & 1 & 6.7 \\
$50-59$ & 0 & 0 \\
$60-69$ & 9 & 14.5 \\
$70-79$ & 13 & 20.0 \\
$80+$ & 15 & 25.0
\end{tabular}

MACROSCOPIC FINDINGS In the main pulmonary arteries the lesions appear as glistening, yellowish white, fibrous, intimal bands crossing the lumina. These have been well illustrated in the paper of Vaněk (1961). In the conducting or elastic intrapulmonary arteries the lesions vary in appearance. In some cases a single or double cord can be seen crossing the lumen (Fig. 1); in others the lumen is filled with a delicate, lace-like network and in a few the lesion appears as a relatively thick but fenestrated, whitish, fibrous membrane. It is impossible to assess the age of the lesions from the gross fixed specimens. In general the lesions appear as white, shining bands of tissue but in some instances they have thrombus adherent to them or they have a reddish hue suggesting the organization of thrombotic material.

HISTOLOGICAL APPEARANCES The fully developed lesions, as seen in the elastic pulmonary arteries, present a typical appearance (Figs. 2, 3, and 4). All changes occur within the internal elastic lamina of the artery and the original lumen may become filled by strands of tissue of varying thickness and com- 


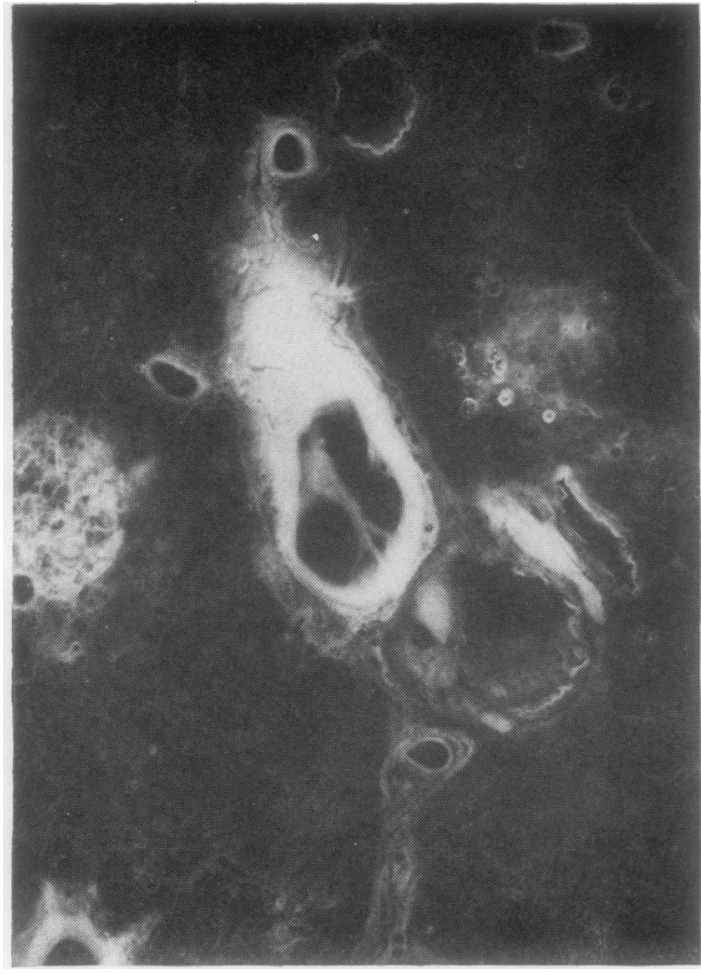

FIG. 1.

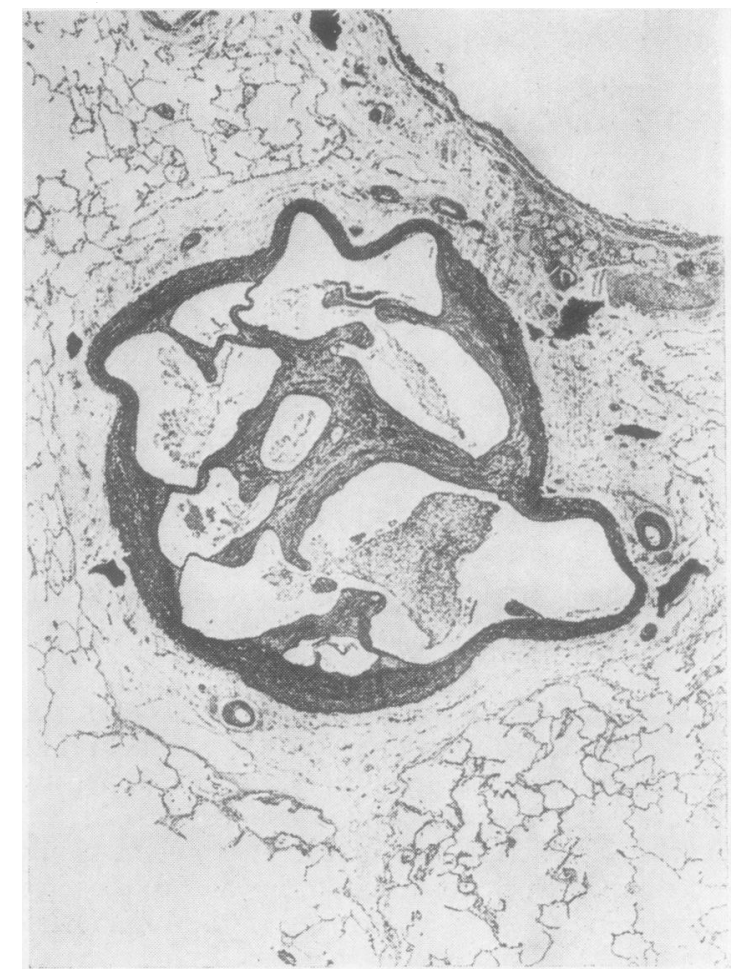

FIG. 2.

FIG. 1. A gross specimen of fixed lung, showing a fibrous band in a conducting pulmonary artery, from a 74-year-old man. Death was due to massive pulmonary embolism. Several previous admissions with left ventricular failure.

FIG. 2. Fully developed fibrous network in a conducting pulmonary artery. Approximately $30 \%$ of the lumen is occluded by the lesion. From a necropsy on an 87-year-old male with previous hospital admissions eight and three years before death. No fresh emboli were found. Masson trichrome $\times 17$.

position. All the channels found within the original arterial lumen are lined by endothelium. The more delicate strands of tissue are composed of collagen with a few fibroblasts. In the stouter bands there is more organization and areas of greater cellularity can be seen with occasional lipophages (Fig. 5). Haemosiderin-laden macrophages are present in some instances but are not usually a prominent feature of the lesions (Fig. 6). Orcein preparations reveal a few rather attenuated and fragmented strands of elastic tissue which never achieve the prominence of true elastic laminae. A most striking feature in some cases is the presence of smooth muscle fibres (Fig. 6) with their typical morphological characteristics of rounded but elongated nuclei and cytoplasm which gives the tinctorial reactions of muscle when exposed to the van Gieson and trichrome stains.

Careful examination of many cases in the whole series, representing thrombi of all ages, shows a spectrum of change from that of the freshly impacted embolus to that of the fully organized bands of fibrous tissue. The first stage after impaction is the covering of the thrombus with endothelial-like cells which also appear to invade it at, or adjacent to, the points of its attachment to the arterial wall and these cells form many new vascular channels within the embolus. A further feature is the vasodilatation of the vasa vasorum at the point of attachment of the embolus to the arterial wall. The vasodilatation is accompanied by oedema of the media of the parent artery. No examples were encountered in which the vasa vasorum could be seen crossing the full thickness of the vessel wall thereby invading the thrombus. The organization of an embolus, including the invasion by fibroblasts with the formation of collagen around the new endothelial-lined channels, and even the formation of smooth muscle, occurs from within the lumen of the parent vessel as in the case of the systemic circulation (Dible, 1958). 


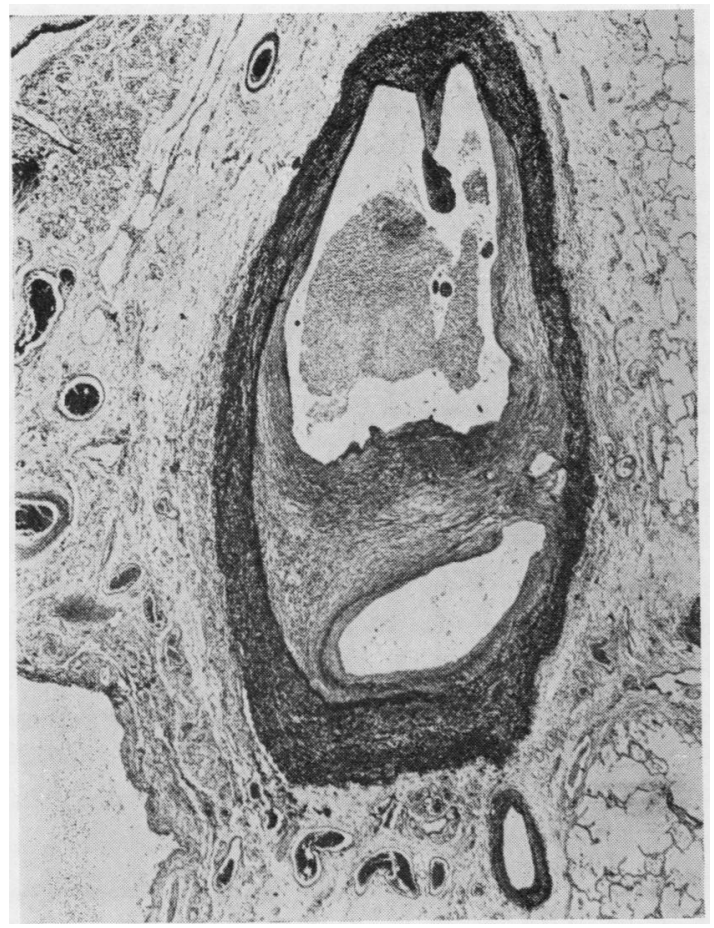

FIG. 3.

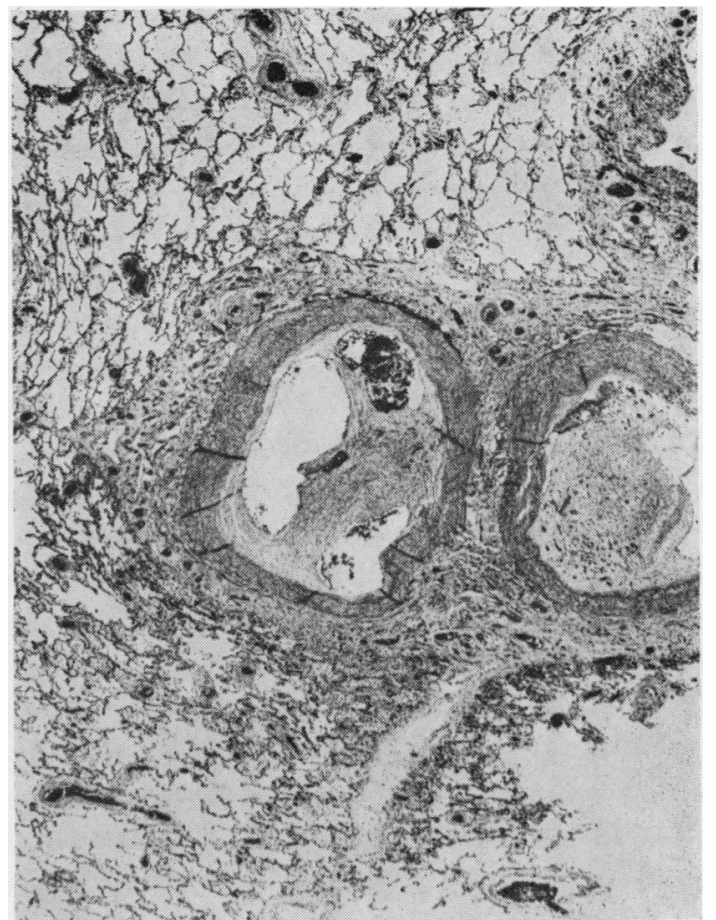

FIG. 4.

FIG. 3. A fibrous band merging with intimal fibrosis. The fibrous tissue inside the internal elastic lamina occupies approximately $40 \%$ of the lumen. A 76-year-old male with hospital admissions ten and eight years before death from coronary thrombosis. No fresh emboli found. Orcein $\times 15$.

FIG. 4. Fibrous bands present in two adjacent conducting arteries, just distal to a bifurcation. The original embolus probably lodged astride the bifurcation. More than $50 \%$ of the lumen of the lower vessel is occluded by fibrous tissue. A 65-year-old male with admissions to hospital five years and two months before death from coronary thrombosis. No fresh embolus found. Masson trichrome $\times 17$.

\section{DISCUSSION}

The presence of fibrous bands in the conducting (elastic) pulmonary arteries is likely to escape detection unless the lung is examined carefully at necropsy. Lesions in the pulmonary trunk or main pulmonary artery may be seen in the fresh specimen but if lesions in the intrapulmonary elastic arteries are to be detected it is imperative that the lung be fixed in the expanded state and then thin slices examined with care. Even when this method is used, lesions contained in the muscular pulmonary arteries may be missed when the lung slices are examined by the unaided eye, and for this reason the incidence of bands and webs found in the muscular pulmonary arteries in this series may be too low. For the purposes of the present paper the study of bands and webs has been confined to those found in the elastic pulmonary arteries.

The first description of fibrous bands in the pul- monary artery is that of Zahn (1889) who described a lace-like structure in the lumen of the left pulmonary artery in a 52-year-old woman who died of a massive pulmonary embolus. The structure was composed of strands of fibrous tissue attached to the intima. Zahn believed it to be congenital, having dismissed the idea of a thrombotic origin. Posselt (1909) described a case with a band in the main pulmonary trunk, the band being attached to a cusp of the pulmonary valve. He also favoured a congenital origin. The first description of similar structures in the smaller elastic intrapulmonary arteries is that of Möller (1922) who reported four cases and was the first to support the theory that the webs were formed from pulmonary emboli, his evidence being the presence of haemosiderin in the bands. Steinberg (1929) gave a similar account of these lesions in the smaller pulmonary vessels. Saphir (1932) reported in detail two instances of fibrous bands and ridges in the pulmonary arteries. 


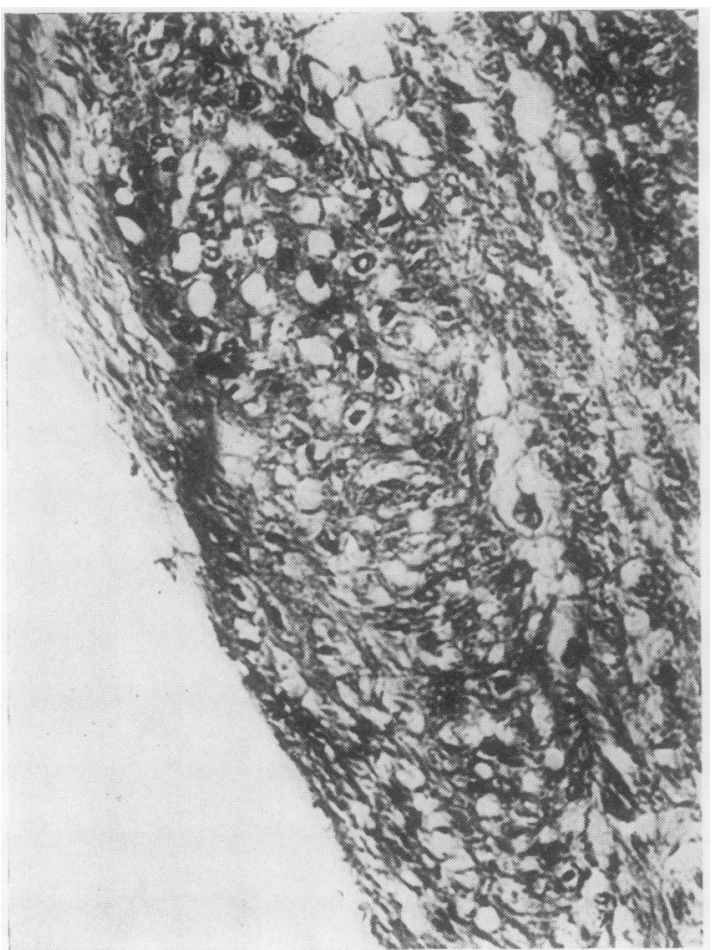

FIG. 5 .

FIG. 5. Higher-power view of part of Figure 2 showing vacuolated, lipid-containing cells in a fibrous band. Masson trichrome $\times 100$.

FIG. 6. A high-power view of a portion of a fibrous band showing some smooth muscle fibres in the subendothelial tissues at the top right hand corner of the photograph. The masses of dark material in the centre and on the right hand side of the photograph are clumps of haemosiderin. Masson trichrome $\times 100$.

In particular he noted that the bands contained fibroblasts, collagen, fibrin undergoing organization, and haemosiderin. He concluded that the bands were organized thrombi and suggested that fibrous intimal thickening or arteriosclerosis found in the smaller pulmonary arteries had the same origin.

Vaněk (1961) studied the major conducting pulmonary arteries by opening them with scissors in fresh unfixed lungs and found fibrous band lesions to be present in $9 \%$ of 1,349 necropsies on individuals over the age of 40 years. In some cases there were also organizing or fresh emboli. In an extensive investigation Korn, Gore, Blenke, and Collins (1962) reported similar structures in 52 cases, $25 \%$ of which died of pulmonary embolic disease. All these later workers were convinced of the embolic origin of the lesions.

Fibrous bands and webs are not the only lesions resulting from organization of pulmonary emboli. Intimal fibrosis may also result but is not considered in the present paper.
The histogenesis of the complex intraluminalo pattern of the fibrous bands probably depends on three factors: (1) retraction or shrinkage of the em-윽 bolus; (2) organization of the embolus, and (3) the original form of the embolus. Retraction of the 3 embolus and fibrinolysis are often stated to play a major part in the fate of pulmonary emboli (Korn et al., 1962; Heard, 1952). This is because in experi- $N$ mental pulmonary embolism carried out with blood clots or fibrin emboli, there is a remarkably rapid disappearance of the emboli occurring within days $\omega$ or even hours of embolization. Allison, Dunnill, and Marshall (1960), using large blood clots aso experimental emboli in eight dogs, found that the material had completely disappeared from the ${ }^{?}$ pulmonary circulation in one of the two dogs $\frac{T}{0}$ allowed to live for four weeks, and there was $\mathrm{a}_{\overrightarrow{\mathrm{D}}}$ fibrous excrescence on the wall of a conducting? vessel in the other dog. When thrombus, formed in $\odot$ the inferior vena cava, was used by Marshall, Sabiston, Allison, Bosman, and Dunnill (1963) the 
organization of the embolus occurred more slowly.

It must be emphasized that the situation in animal experiments is not truly comparable to the naturally occurring situation found in man. Pulmonary embolism is studied as a primary lesion in fit, young animals whereas in man embolism occurs, except in a few cases, as a secondary complication associated with a large range of diseases and is most common in aged, ill patients. The embolic material is also different, consisting in man of ante-mortem thrombus. Factors determining the fate of emboli in man have not been elucidated but it is likely that many factors, for example, the age and composition of the thrombus at the time of embolization, diseases of the lungs and heart, and the balance between lytic and coagulation factors of the blood, are involved.

In emboli which are organized to form networks, the final form of the network may depend, to a greater or lesser extent, on the physical shape of the original embolus. At necropsy fresh pulmonary emboli are frequently seen as coiled masses occluding the pulmonary artery branches, or as elongated thrombi lying astride an arterial bifurcation. The former are most likely to give rise to fibrous bands because the latter are often non-occlusive and undergo organization to form fibrous excrescences. Figure 7 is a longitudinal section of a pulmonary artery occluded by a recent coiled thrombus. In the section the embolus can be seen to consist entirely of laminated ante-mortem thrombus, without any homogeneous propagation clot or invasion of the material by fibrous tissue. If no further retraction of this embolus occurred, but the thrombus was entirely replaced by collagen, its appearance would be typical of a fibrous band lesion.

The fact that these fibrous bands represent one of the end results of pulmonary emboli is of great importance in any attempt to assess the incidence of pulmonary embolism. The incidence of fibrous bands at necropsy is an indication, though it is not known how good an indication, of the incidence of pulmonary embolism in the living.

In only one of the 38 cases was there evidence of past history of pulmonary embolism although in 28 cases there had been previous hospital admissions or a prolonged illness. Perusal of the clinical notes of these admissions has revealed only an otherwise unexplained pyrexia and tachycardia for one or two days in a few cases, as possible evidence of pulmonary embolism.

It must be emphasized, however, that pulmonary embolism may follow a minor illness or injury, which the patient most likely would not mention, and which would not be recorded in the notes.

The majority of embolic episodes are apparently clinically silent, as is illustrated by the following case:

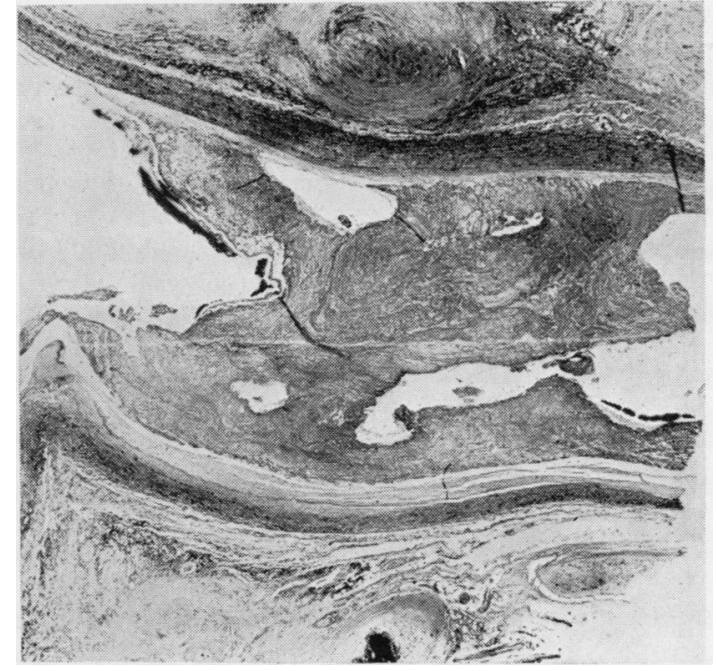

FIG. 7. Longitudinal section of a recent embolus in a conducting pulmonary artery showing appearance of coiled thrombus in section. Haematoxylin and eosin $\times 5$.

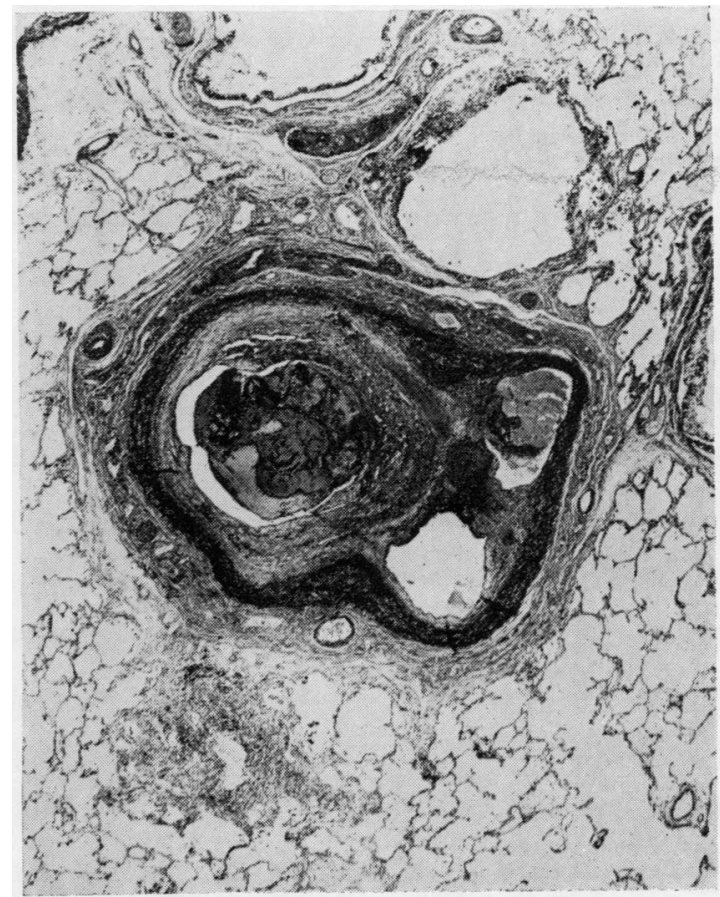

FIG. 8. Conducting pulmonary artery with recent pulmonary embolus superimposed on old fibrous band lesion in a 70-year-old woman with disseminated sclerosis since the age of 24 years. Several previous admissions to hospital. No fresh embolus noted at necropsy in main pulmonary arteries. Masson trichrome $\times 15$. 
A 47-year-old man (R.I. 329757) was admitted to the Radcliffe Infirmary on I July 1965 having been well, apart from the occurrence of a subarachnoid haemorrhage 13 years previously, until an hour or two before admission when he suddenly became unconscious. He died 36 hours later and at necropsy was found to have a cerebral haemorrhage due to rupture of an aneurysm of the right anterior cerebral artery. Examination of the pulmonary arteries revealed fibrous bands in the conducting vessels, possibly a sequel of the subarachnoid haemorrhage, and an organizing thrombus which was of some days' duration and must have been present before the clinical onset of his final illness.

The haemodynamic consequence of a fibrous web is not known but it presumably offers a resistance to blood flow. One or two webs in the conducting pulmonary vessels in a healthy subject are of no significance but there have been many cases recorded of cor pulmonale due entirely to multiple recurrent emboli forming bands and webs, the last embolus being fatal (Belt, 1939; Castleman and Bland, 1946; Irvin, 1949). The significance of pulmonary emboli probably depends on the reserve of lung tissue. In a patient whose lungs have been compromised, for example by parenchymal disease, congestion, or previous pulmonary emboli, to such an extent that there is minimal or no reserve, the occurrence of an embolus may prove fatal whereas in a healthy subject the embolus may occur unnoticed.
We are grateful to Mrs. Madeline Creighton for technical assistance. One of us (M.S.D.) is in receipt of a personal grant from the Medical Research Council.

\section{ADDENDUM}

Since submitting this article we have had the opportunity of studying Virchow's Gesammette Abhandlungen $z u$ Wissenschaftlichen Medicin (1856) and must now attribute the first description of $\vec{\circ}$ fibrous bands and webs to him. He was familiar with these lesions in pulmonary arteries and recognized that the fibrous bands and webs originated from pulmonary emboli.

\section{REFERENCES}

Allison, P. R., Dunnill, M. S., and Marshall, R. (1960). Thorax, 15, 273.

Belt, T. H. (1939). Lancet, 2, 730.

Castleman, B., and Bland, E. F. (1946). Arch. Path., 42, 581.

Dible, J. H. (1958). J. Path. Bact., 75, 1.

Heard, B. E. (1952). Ibid., 64, 13.

Irvin, G. E. (1949). Amer. Heart J., 37, 1144.

Korn, D., Gore, I., Blenke, A., and Collins, D. P. (1962). Amer. J. Path., 40, 129.

Marshall, R., Sabiston, D. C., Allison, P. R., Bosman, A. R., and Dunnill, M. S. (1963). Thorax, 18, 1 .

Möller, P. (1922). Beitr. path. Anat., 71, 27.

Posselt, A. (1909). Ergebn. allg. Path. path. Anat., 13, 298.

Saphir, O. (1932). Arch. Path., 14, 10.

Steinberg, U. (1929). Beitr. path. Anat., 82, 443.

Vaněk, J. (1961). J. Path. Bact., 81, 537.

Virchow, R. (1856). Gessammette Abhandlungen zu Wissenschaftlichen Medcin, p. 219. Frankfurt A.M.

Zahn, F. W. (1889). Virchows Arch. path. Anat., 115, 58. 